\title{
Seasonal Variation of the Effect of Extremely Diluted Agitated Gibberellic Acid (10e-30) on Wheat Stalk Growth: A Multiresearcher Study
}

Peter Christian Endler, Wolfgang Matzer, Christian Reich, Thomas Reischl, Anna Maria Hartmann, Karin Thieves, Andrea Pfleger, ürgen Hoföcker, Harald Lothaller, and Waltraud Scherer-Pongratz

Division Complementary Health Sciences, Interuniversity College for Health and Development Graz, Castle of Seggau, 8042 Graz, Austria

Received 21 March 2011; Revised 9 August 2011; Accepted 9 August 2011

Academic Editor: Joav Merrick

The influence of a homeopathic high dilution of gibberellic acid on wheat growth was studied at different seasons of the year. Seedlings were allowed to develop under standardized conditions for 7 days; plants were harvested and stalk lengths were measured. The data obtained confirm previous findings, that ultrahigh diluted potentized gibberellic acid affects stalk growth. Furthermore, the outcome of the study suggests that experiments utilizing the bioassay presented should best be performed in autumn season. In winter and spring, respectively, no reliable effects were found.

KEYWORDS: homeopathy, ultra high dilution, bio-assay, gibberellic acid, wheat 


\section{INTRODUCTION}

The bioassay on wheat stalk growth has been used in studies on homeopathy for decades, originally using homeopathically prepared (potentized) metal salts [1]. An inhibition of growth by silver nitrate diluted above Avogadro's value was found by 3 of 4 researchers (Kolisko, Scherer-Pongratz, Nograsek, but not Endler) [2, 3]. Pfleger et al. reported an inhibition of wheat growth by high diluted gibberellic acid [4]. Betti et al. [5] and Brizzi et al. [6] reported a stimulation of wheat growth through treatment of the seeds with high potencies of arsenic. On replicating the experiment however, Binder et al. [7] found a significant decrease in longitudinal growth. It is interesting to note that in these cases, data were usually found to be homogeneous within groups [8]. This has led to the idea that calculation on the basis of absolute differences between means of verum and control group may be a useful statistical tool complementing calculation of means alone [9].

For the study presented here, the use of ultrahigh diluted potentized hormones has been inspired by zoological studies (on amphibians and thyroxin) [10-12] and by botanical studies of Baumgartner et al. (dwarf pea shoot growth/duckweed development and gibberellic acid) [13-15]. Baumgartner's experiments showed a reproducible stimulation of growth by the dilution $10^{-17}$ in some but not all subexperiments, depending on the harvest lots used. Homeopathically prepared gibberellic acid was also tested on barley stalk length, with different results according to seedlings' vigour levels [16].

The aim of the study presented here was to test the influence of an extreme dilution of gibberellic acid $\left(10^{-30}, 30 \mathrm{x}\right)$ prepared according to a protocol derived from homeopathy on wheat germination and stalk length after one week. Following up on pilot experiments which had yielded observable effects the authors performed control experiments at different seasons of the year. The research question was as follows: does treatment with gibberellic acid 30x result in altered germination behaviour and/or stalk growth of wheat seedlings, measured after 7 days, when compared with analogously prepared solvent?

First results from experiments performed in autumn 2007 (see Table 1, experiments A1-4) suggested an inhibition of stalk growth by gibberellic acid 30x [4]. Further experiments (Table 1, WS1-4) led to the idea that gibberellic acid 30x causes inhibition of growth in autumn season only, whereas in winter it causes stimulation of growth [9]. To investigate the hypothesis of seasonal dependency, further experiments (A5-9, WS5) were performed and all data were submitted to a comprehensive analysis.

\section{METHODS}

In preparing the documentation of the experiments, the recommendations for good fundamental research documentation in homeopathy were observed, which were elaborated by the K. and V. Carstens Foundation, Essen [17].

\subsection{Plants}

Experiments were performed on wheat (Triticum aestivum, Capo variety, procured from Gosch organic farming, Aibl, Austria) grain grown without herbicides or pesticides (harvest 2007, 2008, and 2009). Around $10 \%$ of the grains were ruptured and around $10 \%$ were distorted, and these were all removed prior to the experiment.

\subsection{Researchers and Sites (Interresearcher Control): Season}

All autumn experiments were performed at the laboratory of the Interuniversity College in Weiz near Graz, by 5 different researchers (see Table 1). Winter/spring experiments were performed at different locations (Table 1), by 4 researchers. Laboratory workers both received thorough training in the methods and procedures to be used (Scherer and Endler). They had no contact with each other while experiments were in progress. The project was coordinated by Endler. 
TABLE 1: Overview of 7-day experiments on wheat stalk growth under the influence of potentized gibberellic acid (G30x) versus analogously potentized solvent (W30x) carried out at the Interuniversity College in the time from 2007 to 2010. Work is shown subdivided into batches of ca. 500 grains G30x and W30x each, referred to in the following as experiments. Altogether there were 15 such experiments, performed by 8 researchers. Year and month: time of the experiment; lab.: laboratory in which the experiment was carried out; pot: person preparing the potencies; acet.: whether the mother tincture (for both G30x and W30x) contained acetone; age: age of the wheat at the time of the experiment in years; dishes: number of germination dishes per group (dishes contained 20 or 25 grains each, depending on the experiment, see Table 3). In italics: pilot experiments [4].

\begin{tabular}{lcccccccc}
\hline No. & Researcher & Year & Month & Lab. & Pot. & Acet. & Age & Dishes \\
\hline A1 & Pfleger & 2007 & Oct & Weiz & Pfleger & yes & 0 & 25 \\
A2 & Pfleger & 2007 & Oct & Weiz & Pfleger & yes & 0 & 25 \\
A3 & Hoföcker & 2007 & Dec & Weiz & Hoföcker & yes & 0 & 25 \\
A4 & Hoföcker & 2007 & Nov & Weiz & Hoföcker & yes & 0 & 22 \\
A5 & Reich & 2008 & Dec & Weiz & Reich & yes & 0 & 20 \\
A6 & Hartmann & 2009 & Sep & Weiz & Scherer & no & 0 & 25 \\
A7 & Scherer & 2009 & Oct & Weiz & Scherer & no & 0 & 25 \\
A8 & Scherer & 2009 & Dec & Weiz & Scherer & no & 0 & 25 \\
A9 & Scherer & 2009 & Dec & Weiz & Scherer & no & 0 & 25 \\
WS1 & Reischl & 2009 & Jan & Weiz & Reischl & yes & 1.5 & 20 \\
WS2 & Thieves & 2009 & Jan & Gels. & Reich & yes & 0.5 & 25 \\
WS3 & Thieves & 2009 & Jan & Gels. & Reich & yes & 0.5 & 25 \\
WS4 & Pfleger & 2009 & Feb & St.Jo & Pfleger & no & 0.5 & 32 \\
WS5 & Matzer & 2010 & Feb & Weiz & Scherer & no & 0.5 & 25 \\
WS6 & Pfleger & 2008 & Apr & St.Jo & Pfleger & yes & 0.5 & 25 \\
\hline
\end{tabular}

\subsection{Laboratory Conditions}

All glass bottles and fastenings were disposable products; dishes, covering glass vessels, and glass pipettes for administration of the probes were heat-sterilised and were (additionally) rinsed twice with double distilled water prior to treatment. Plastic pipettes used for the dilution process were disposable products. Seedling development took place in complete darkness at a temperature of $21.5 \pm 1{ }^{\circ} \mathrm{C}$ depending on the laboratory.

\subsection{Preparation of Test Solutions}

The test substance and control were prepared and inspired by Baumgartner et al. [13] according to the method of stepwise dilution and succession as derived from homeopathy. The degree of dilution was set to $10^{-30}$ in order to exceed Avogadro's limit of theoretical 0 molarity $\left(10^{-24}\right)$. Botanic hormone $10^{-30}$ (30x) was chosen with regard to previous experiments with a zoological hormone 30x [10-12]. Grains were observed under the influence of gibberellic acid 30x, or of analogously prepared water control (30x), respectively. Different sets of test substance and control, respectively, were prepared by different researchers (see Table 1).

For preparation of the test dilutions, $0.017 \mathrm{~g}$ of gibberellic acid (Sigma-Aldrich company, art. no. 36575 ) were either added to $9 \mathrm{~mL}$ of acetone or to $9 \mathrm{~mL}$ of double distilled water (see Table 1) and the liquid was gently swung (not "agitated") for one minute (= "mother substance, 1x"). Then, using a disposable pipette (Brand company, Transferpette $100 \mu$ ), $1 \mathrm{~mL}$ of the mother substance was added to $9 \mathrm{~mL}$ of double distilled water in a $20 \mathrm{~mL}$ brown glass bottle (Heiland company, art. no. 380020) and the product was 
agitated vigorously according to a standardized protocol: the vial was manually banged 30 times against an elastic surface at intervals of approximately $2 \mathrm{~s}$ to create mechanical shocks (= "gibberellin $2 \mathrm{x}$ "). In a total of 30 steps of dilution 1:10 and 29 steps of agitation (as agitation was omitted at the first dilution step), the test substance "gibberellin 30x" was thus prepared. Starting from the 28th step, quantities larger than $1 \mathrm{~mL}$ were added to the tenfold amount of double distilled water in order to prepare a sufficient quantity of test substance. Larger brown glass bottles (each of which was filled $1 / 2$ with the liquid) were used for these last steps (29x: $250 \mathrm{~mL}, 30 \mathrm{x}: 500 \mathrm{~mL}$ ). A new glass bottle was used at each step of dilution.

Analogously prepared solvent (i.e., in 1x either acetone or water (see Table 1), then water in steps $2 \mathrm{x}$ to 30x) was used for control (water 30x) to ensure that possibly solute contents of the glass wall were equally present both in verum 30x and control 30x and thus their possible effect was ruled out, and that the content of solute oxygen was alike. If a difference in growth occurred between seedlings treated with verum and control, it should then be due to the presence or absence of gibberellic acid in the mother substance.

Table 1 gives an overview of experiments performed grouped by time of season (A1-A9 in autumn, WS1-WS6 in winter or spring).

\subsection{System Performance Controls}

Experiments have shown that differential treatment with water 30x or with water that has not undergone any preparation process at all $\left(\mathrm{W}^{\circ}\right.$, negative control) produces no differences in stalk length measured after one week (water 30x: $49.7 \pm 21.6 \mathrm{~mm} ; \mathrm{W}^{\circ}: 49.9 \pm 21.24 \mathrm{~mm}$ ). In these experiments, $N$ of grains per group was 2000 , and temperature was $21.5 \pm 1^{\circ} \mathrm{C}$.

By way of a positive system control it has been observed that after one week stalk lengths are greater under treatment with gibberellic acid at molecular doses $\left(10^{-4}: 53.8 \pm 22.1 \mathrm{~mm} ; 10^{-6}: 46.9 \pm 22.5 \mathrm{~mm}\right)$ than in water control $(44.8 \pm 22.6 \mathrm{~mm})\left(N\right.$ of grains per group $=200$, temperature $\left.20 \pm 1^{\circ} \mathrm{C}\right)$.

Analyses of water control in analogous experiments in the past with the same spatial arrangement of dishes and plants have shown a high degree of homogeneity within dishes of one and the same group. Homogeneity is also investigated in the present study.

\subsection{Independent Probe Coding}

Control and verum were encoded by further independent authorities. All probes were applied blindly, codes were broken only after the data had been calculated.

\subsection{Data Base}

Two sets of 217 dishes for treatment with verum and with control, respectively, were used for the autumn experiments. Depending on the researcher, 20 or 25 grains (see Table 3) were put into one dish, that is, 4,440 grains were observed per treatment group.

For the experiments performed in winter/spring, two sets of 152 dishes were used, 3,140 grains were observed per treatment group.

\subsection{Placement of Grains}

The grains were put into glass dishes (diameter $11 \mathrm{~cm}$ ), each containing 2 layers of filter paper (Whatman, cellulose, $90 \mathrm{~mm}$, sort 2), with the germination furrow facing down (Figure 1).

\subsection{Exposition to Probes}

$5 \mathrm{~mL}$ of the verum or control probe were added to each dish with the help of a disposable $5 \mathrm{~mL}$ pipette and pipetting ball (VWR company, art. no. 612-1328 and 612-1947). Dishes were then covered with $1000 \mathrm{~mL}$ glass vessels and dishes and covers were wrapped in aluminium foil (see Figure 2). 


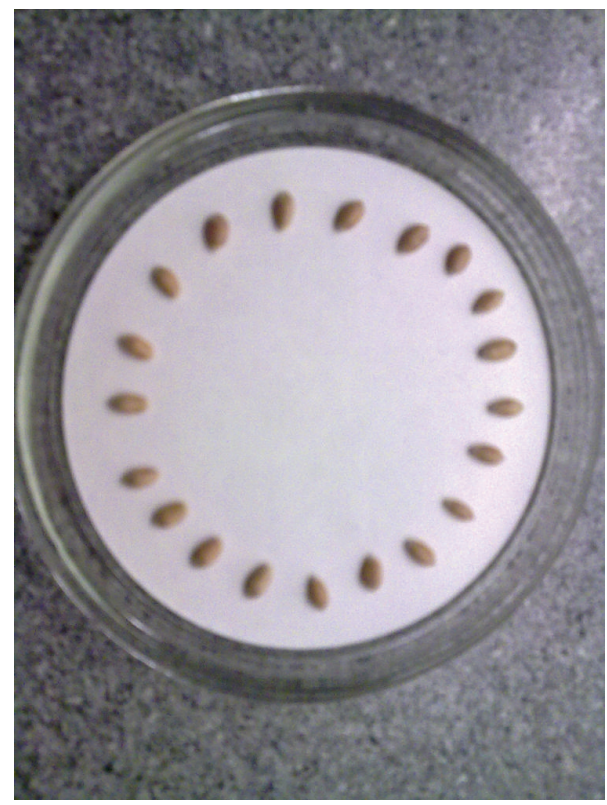

FIGURE 1: Example for placement of grains.

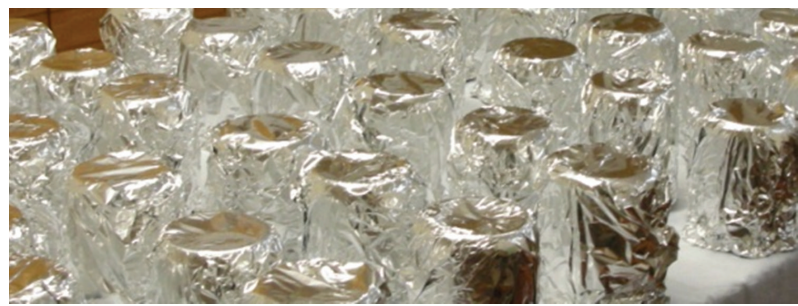

FIGURE 2: Example for placement of beakers.

They were placed in alternating rows according to a random procedure (stratified randomisation). Grains had not been soaked prior to treatment.

\subsection{Observed Development (Endpoints)}

Germination and stalk length (Figure 3) were observed after 7 days according to standard protocol [2]. Stalks were cut off and measured by naked eye on a mm scale. The person performing the measurements knew neither whether the stalks measured were verum- or control-treated (see blinding procedure above) nor what their blind code (A or B) was. Any possibility of an assignment bias was thus ruled out. Subsets were harvested in the same sequence as they had been planted. Measurement of endpoints was done blindly.

\subsection{Data Evaluation}

The number of germinated seedlings was compared with the number of nongerminated seedlings in both the verum and the control group in a four-field table according to the chi-square test.

For description of stalk length, at the level of the 15 individual experiments, the statistical mean was used, and lengths were compared by one way analysis of variance. S.D. of the mean was calculated. Mean and S.D. were also calculated by dish, that is, for each cohort of 20 or 25 grains. In order to avoid false negative results, analysis of variance was not calculated at dish level, and to avoid false positive results, it 


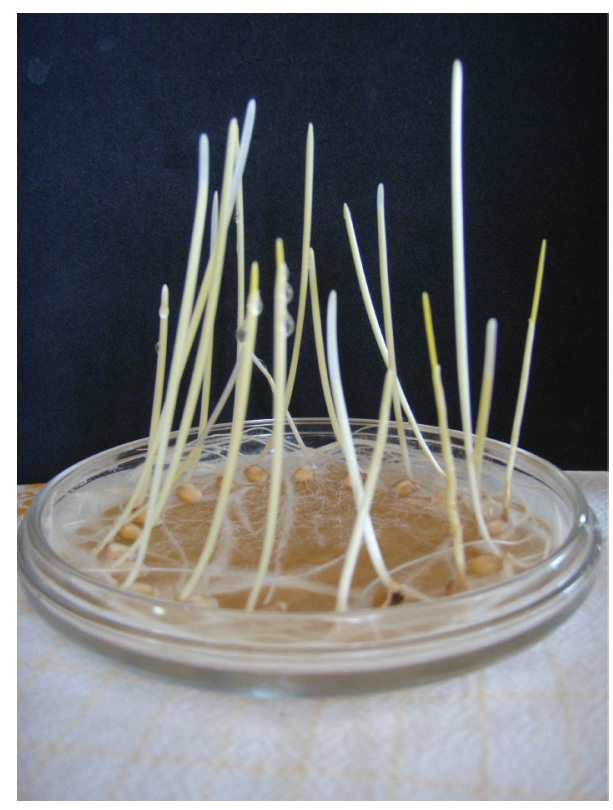

FIGURE 3: Example of stalk growth, from [4].

was also not calculated for the pooled experiments. For the pooled experiments, however, the effect size (Cohen's d, standardized difference of means $=$ absolute difference between means of verum and control group, divided by S.D.) was calculated. An effect size $>0.2$ is regarded as small, $>0.5$ as medium, and $>0.8$ as large.

Homogeneities of stalk lengths within the verum group and within the control group, respectively, were investigated by one way analyses of variance with post hoc pairwise comparisons by means of Tukey HSD test.

For control of interrater reliability, the interaction between "treatment" (verum versus control) and "experiments" was calculated by univariate two-way analyses of variance.

Evaluation of data was done blindly, that is, the statistician (Lothaller) was not aware of the meaning of the codes used. Codes were broken only after calculation of results.

Results at the level of single experiments were represented graphically by zeroing the results of the W30x control groups and plotting the difference to the G30x groups on the ordinate. Results at dish level were calculated by dish pair and ordered according the joint mean stalk length (arithmetic mean of mean stalk length in the W30 dish and mean stalk length in the G30x dish assigned to it in the floorplan), and the pairwise differences between means were plotted on the ordinate.

\section{RESULTS}

Germination rates after 7 days were slightly higher for the autumn experiments (96.1\%) than for the winter/ spring experiments $(94.8 \%)(P>0.05)$, with a nonsignificant trend of more seedlings having germinated in the verum group in the autumn experiments $(P>0.05)$ (see Table 2).

With regard to stalk growth, Table 3 and Figures 4 and 5 give an overview of the 15 experiments.

Variability was naturally lower at dish level than it was at grain level.

Figure 4 shows the differences between the mean stalk length of G30x and W30x seedlings (nongeminated grains not considered). As can be seen, all of the 9 autumn experiments on stalk length (i.e., pilot as well as repetition experiments) showed shorter stalks in the G30x group after 7 days. The difference is significant with $P<0.01$ for experiments 1,3 , 4, and 8 , with $P<0.05$ for experiments 2,5 , and 9 , and non-significant $(P>0.05)$ for experiments 6 and 7 . 
TABLE 2: Germination rates of wheat after 7 days under the influence of extremely diluted agitated gibberellic acid (30x) and control. For details, see Table 1.

\begin{tabular}{lcc}
\hline Series $(N)$ & G30x $(\%)$ & W 30x $(\%)$ \\
\hline All autumn exp. & 96.6 & 95.6 \\
All winter/spring exp. & 94.8 & 94.8 \\
\hline
\end{tabular}

TABLE 3: Overview of results of stalk length measurement in the experiments listed in Table 1, each shown for "all grains": all treated grains (upper line) and "germinated grains only": only those grains which germinated during the 7 days of the experiment (lower line). Mean W30x: mean stalk length in the W30 group (in mm); mean G30x: mean stalk length in the G30x group; S.D.: standard deviations: "grains": at grain level (i.e., S.D. of 500 values), "dishes": at dish level (i.e., S.D. of 20 or 25 values). P: significance level of differences between groups at grain level and dish level.

\begin{tabular}{|c|c|c|c|c|c|c|c|c|c|c|}
\hline & Set & Grains & $\begin{array}{l}\text { mean } \\
W 30 x\end{array}$ & $\begin{array}{l}\text { mean } \\
G 30 x\end{array}$ & $\begin{array}{l}\text { S.D. } \\
\text { Grains } \\
W 30 x\end{array}$ & $\begin{array}{l}\text { S.D. } \\
\text { Grains } \\
\text { G30x }\end{array}$ & $\begin{array}{c}P \\
\text { grains } \\
\text { G30x:W30x }\end{array}$ & $\begin{array}{l}\text { S.D. } \\
\text { dishes } \\
W 30 x\end{array}$ & $\begin{array}{l}\text { S.D. } \\
\text { dishes } \\
\text { G30x }\end{array}$ & $\begin{array}{c}P \\
\text { dishes } \\
\text { G30x: W30x }\end{array}$ \\
\hline \multirow{2}{*}{$A 1$} & All & $500+500$ & 47.13 & 42.50 & 20.31 & 21.13 & 0.010 & 3.11 & 3.62 & $<.001$ \\
\hline & germ. & $488+482$ & 48.28 & 44.09 & 19.14 & 19.82 & 0.001 & 3.33 & 3.6 & $<.001$ \\
\hline \multirow{2}{*}{$A 2$} & All & $500+500$ & 44.02 & 42.50 & 20.2 & 19.8 & 0.264 & 3.22 & 4.05 & 0.149 \\
\hline & germ. & $480+493$ & 45.85 & 43.10 & 18.47 & 19.27 & 0.023 & 3.04 & 4.13 & 0.011 \\
\hline \multirow{2}{*}{ A3 } & All & $500+500$ & 45.6 & 40.68 & 24.23 & 23.4 & 0.001 & 8.1 & 9.95 & 0.061 \\
\hline & germ. & $478+476$ & 47.7 & 42.73 & 22.66 & 22.07 & 0.001 & 8.9 & 9.84 & 0.094 \\
\hline \multirow{2}{*}{ A4 } & All & $440+440$ & 43.84 & 38.75 & 21.79 & 20.56 & $<.001$ & 5.13 & 3.51 & $<.001$ \\
\hline & germ. & $424+432$ & 45.49 & 39.47 & 20.43 & 20.05 & $<.001$ & 4.66 & 3.75 & $<.001$ \\
\hline \multirow{2}{*}{ A5 } & All & $500+500$ & 57.01 & 53.48 & 20.34 & 19.23 & 0.011 & 4.69 & 3.67 & 0.091 \\
\hline & germ. & $466+460$ & 61.97 & 57.38 & 11.9 & 13.13 & 0.015 & 3.31 & 2.46 & 0.242 \\
\hline \multirow{2}{*}{ A6 } & All & $500+500$ & 50.93 & 50.03 & 20.48 & 21.53 & 0.497 & 4.55 & 5.28 & 0.520 \\
\hline & germ. & $482+488$ & 52.83 & 51.26 & 18.28 & 20.29 & 0.205 & 3.9 & 4.98 & 0.213 \\
\hline \multirow{2}{*}{ A7 } & All & & 49.02 & 46.74 & 21.27 & 21.25 & 0.091 & 4.77 & 6.34 & 0.158 \\
\hline & germ. & $500+500$ & 49.91 & 47.79 & 20.39 & 20.28 & 0.103 & 4.63 & 5.97 & 0.161 \\
\hline \multirow{2}{*}{ A8 } & All & $500+500$ & 49.96 & 46.47 & 22.74 & 22.45 & 0.015 & 6.75 & 5.45 & 0.050 \\
\hline & germ. & $477+485$ & 52.47 & 48.11 & 20.27 & 21.04 & 0.001 & 6.67 & 4.38 & 0.015 \\
\hline \multirow{2}{*}{ A9 } & All & $500+500$ & 48.99 & 46.29 & 21.97 & 23.34 & 0.060 & 6.83 & 6.21 & 0.150 \\
\hline & germ. & $476+477$ & 51.36 & 48.32 & 19.61 & 21.69 & 0.023 & 5.91 & 5.64 & 0.151 \\
\hline \multirow{2}{*}{ WS1 } & All & $500+500$ & 53.62 & 57.62 & 20.17 & 22.43 & 0.001 & 4.61 & 4.19 & 0.070 \\
\hline & germ. & $478+480$ & 56.21 & 60.75 & 16.77 & 18.45 & $<.001$ & 3.96 & 3.49 & $<.001$ \\
\hline \multirow{2}{*}{ WS2 } & All & $500+500$ & 52.34 & 57.39 & 17.46 & 20.27 & 0.001 & 3.86 & 6.61 & 0.002 \\
\hline & germ. & $486+478$ & 53.85 & 59.91 & 15.24 & 16.66 & $<.001$ & 3.4 & 6.16 & 0.013 \\
\hline \multirow{2}{*}{ WS3 } & All & $500+500$ & 54.18 & 54.38 & 21.16 & 19.27 & 0.999 & 5.56 & 4.93 & 0.901 \\
\hline & germ. & $454+467$ & 59.67 & 58.11 & 12.84 & 13.41 & 0.071 & 4.26 & 3.54 & 0.399 \\
\hline \multirow{2}{*}{ WS4 } & All & $640+640$ & 50.41 & 55.45 & 15.60 & 17.23 & 0.001 & 6.56 & 8.27 & 0.009 \\
\hline & germ. & $620+623$ & 52.04 & 56.96 & 12.90 & 14.79 & $<.001$ & 7.13 & 8.72 & 0.008 \\
\hline \multirow{2}{*}{ WS5 } & All & $500+500$ & 47.84 & 46.21 & 13.36 & 13.7 & 0.185 & 2.46 & 4.21 & 0.233 \\
\hline & germ. & $478+483$ & 50.05 & 47.84 & 8.73 & 10.79 & 0.005 & 2.21 & 4.36 & 0.059 \\
\hline \multirow{2}{*}{ WS6 } & All & $500+500$ & 41.02 & 38.49 & 18.2 & 19.39 & 0.069 & 3.6 & 4.5 & 0.033 \\
\hline & germ. & $450+461$ & 44.49 & 42.76 & 14.3 & 15.32 & 0.063 & 3.1 & 4.68 & 0.127 \\
\hline
\end{tabular}

When all the autumn experiments were pooled, mean stalk lengths $(\mathrm{mm})$ were $46.97 \pm 20.50$ for the verum group and $50.66 \pm 19.77$ for control (mean \pm S.D.) at grain level ( $N=4.440$ per group) and \pm 3.87 and \pm 3.38 , respectively, at dish level (217 cohorts of 20 or 25 grains per treatment group). In other words, verum stalk length $(92.72 \%)$ was $7.28 \%$ smaller than control stalk length $(100 \%)$. The effect size is small when calculation is done on the basis of grains $(d=0.18)$ but, due to the smaller S.D. at dish level, high when done on the basis of dishes $(d=1.02)$.

In contrast, no reliable effect was found in experiments performed in winter/spring, as is seen in Figure 5. In 3 experiments G30x seedlings grew longer than W30x seedlings $(P<0.01)$, in 2 experiments 


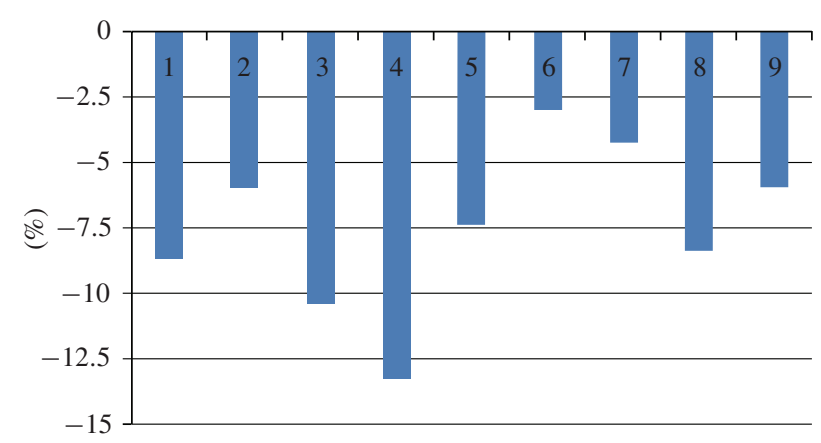

FIGURE 4: Relative differences in stalk length between W30x groups (zeroed) and G30x groups in per cent (ordinate). 1-9: experiments carried out during autumn. For further explanations see text.

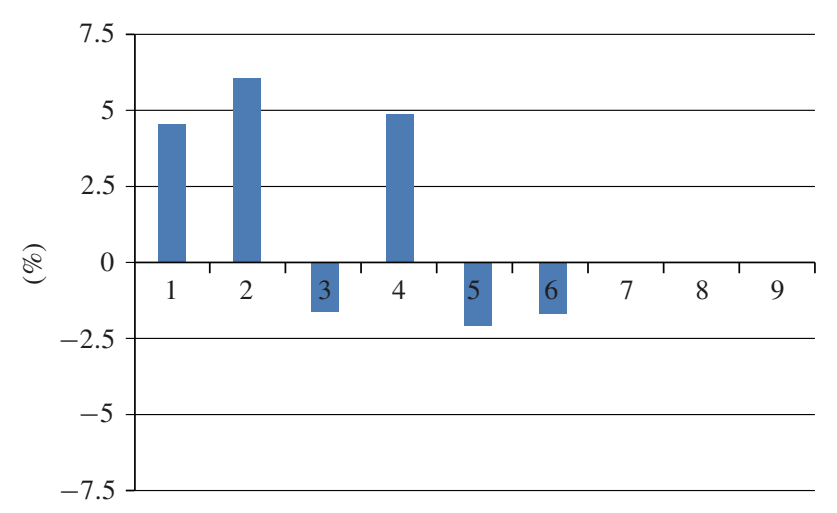

FIGURE 5: Stalk growth in the winter/spring experiments. For further explanations see Figure 4 and text.

there was no significant difference $(P>0.05)$, and in one experiment they grew shorter than W30x seedlings $(P<0.01)$.

When all winter/spring experiments were pooled, mean stalk lengths $(\mathrm{mm})$ were $54.60 \pm 16.41$ for the verum group and $52.68 \pm 14.41$ for control at grain level $(N=3,140$ per group $)$ and \pm 4.93 and \pm 3.59 , respectively, at dish level (152 cohorts of 20 or 25 grains per treatment group), that is, overall verum stalk length $(103.64 \%)$ was $3.64 \%$ greater than control stalk length $(100 \%)$. The effect size is small both when calculation is done on the basis of grains $(d=0.13)$ and on the basis of dishes $(d=0.45)$.

These results suggest that in the experiments performed in autumn, there was a growth inhibiting influence of gibberellic acid 30x. In contrast, no clear effect was found in experiments performed in winter/spring. This points to autumn as the most promising season for obtaining significant effects.

As a rule, data were found to be homogeneous within the control groups of the single experiments $(P>0.05)$ as well as within the verum groups $(P>0.05)$. In other words, there are significant differences between the average stalk lengths between the groups (verum or control, see above), but no significant differences within the groups. This holds true both for the experiments performed in autumn and in winter/ spring.

Figure 6 shows mean stalk length of W30x and G30x seedlings by dish pair (e.g., $25+25$ grains), with dish pairs ordered according to their joint mean stalk length. It illustrates that the difference between G30x and W30x seedlings within dish pairs in the autumn experiments did not depend on absolute stalk length (ranging from ca. $65 \mathrm{~cm}$ on the far left to ca. $35 \mathrm{~cm}$ on the far right). The G30x curve is mostly lower than the W30x curve. 


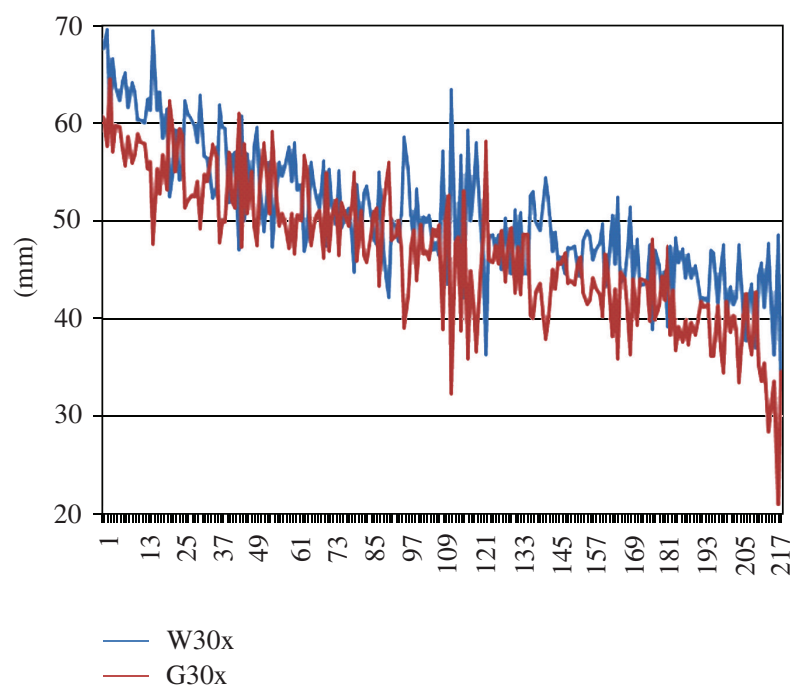

FIGURE 6: Mean stalk length of G30x and W30x seedlings by dish pair in the autumn experiments (A1 through A9), with dish pairs ordered according to their joint mean stalk length. Red line: curve connecting G30x values; blue line: curve connecting W30x values; ordinate: $\mathrm{mm}$; abscissa: dish pairs ordered according to their joint mean stalk length. For further explanations see text.

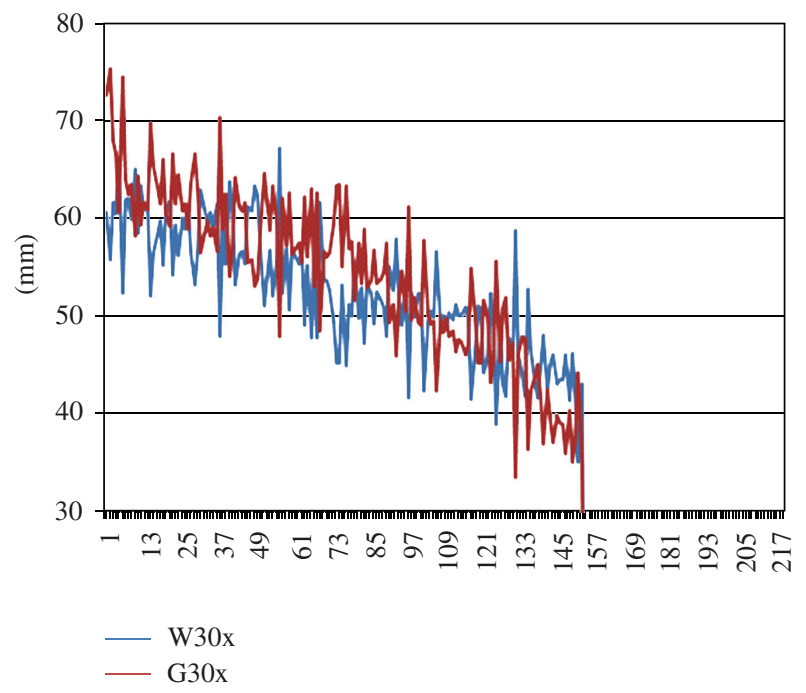

FIGURE 7: Stalk length of G30x and W30x seedlings by dish pair in the winter/spring experiments (WS1 through WS6). For explanations see Figure 6.

In the winter/spring experiments (Figure 7), G30x seedlings showed a trend of growing higher than W30x seedlings towards the high end of the growth range, whereas towards the low end of the growth range there appears to be no clear-cut difference.

\section{DISCUSSION}

First results from experiments performed in autumn 2007 had suggested an inhibition of stalk growth by gibberellic acid 30x [4]. Further experiments then led to the idea that gibberellic acid 30x causes inhibition of growth in autumn season only, whereas in winter it causes stimulation of growth [9]. To investigate 
the hypothesis of seasonal dependency, further experiments were now performed and all data were submitted to a comprehensive analysis.

All of the 9 autumn experiments 2007-2009 (i.e., pilot as well as control experiments) showed less stalk growth in the G30x-group (statistically significant with $P<0.01$ in 4, with $P<0.05$ in 3 cases, trend in 2 cases). Mean stalk lengths $(\mathrm{mm})$ were $46.97 \pm 20.50$ for the G30x-group and $50.66 \pm 19.77$ for control (mean \pm S.D.) at grain level $(N=4,440$ per group) and \pm 3.87 and \pm 3.38 ( \pm S.D.), respectively, at dish level (217 cohorts of 20 or 25 grains per treatment group). In other words, in autumn experiments, verum stalk length $(92.72 \%)$ was $7.28 \%$ smaller than control stalk length $(100 \%)$. The effect size, calculated on the basis of dishes, was high $(d=1.02)$.

In contrast, no clear effect was found in experiments performed in winter/spring (less stalk growth in the verum group in one case, no difference in one case, and more growth in 3 cases). Overall verum stalk length $(103.64 \%)$ was slightly greater than control stalk length $(100 \%)$. The result of the winter/spring experiments could be interpreted as a slight enhancement of growth, that is, effects of G30x would then be contradictory in autumn and in winter.

The working hypothesis, derived from $[4,9]$, was that the time of season (autumn versus winter/ spring) is a crucial factor in predicting the effect of homeopathically prepared gibberellic acid (G30x). This hypothesis, reflected in the arrangement of Table 1, appears to have been confirmed by the present results. Other parameters such as the researcher and the person preparing the dilutions involved, the year the experiment was carried out, and the presence or absence of acetone in the mother substance $1 \mathrm{x}$ did not seem to play a role with regard to the outcome. However, the following factors may also play a key role: the age of the grains (a few weeks in the autumn experiments, as opposed to 0.5 to 1.5 years in the winter/spring experiments, with differences possibly attributable to growth inhibition by G30x in fresh seeds), as well as the laboratory (Weiz in southern Austria versus Sankt Johann in northern Austria and Geilenkirchen in Germany). These factors, as well as a possible influence of slight temperature differences between the experiments, require further investigation.

From a methodological viewpoint it would of course be interesting to have one person perform a series of such experiments in a single laboratory under maximally calibrated conditions. This would sharpen the conclusions of the study. On the other hand, the multicentered approach chosen allows greater generalisability of outcomes.

Other multicentred botanical studies on dilutions above Avogadro's limit can be found in literature, namely, on algae and copper sulphate (growth stimulation of poisoned algae in the initial study only, but not in the repetition), wheat and silver nitrate (increase of stalk growth in 4 out of 5 studies), arsenic poisoned wheat, and arsenicum album (growth stimulation in 2 studies, decrease of growth in 2 studies) [18, 19]. With regard to these studies, the authors can at present say that the model with wheat and gibberellic acid seems to be a promising candidate for a sequence of research projects.

The model may be useful for further research as there exists a theoretical justification due to previous studies with wheat [1-4], as well as with potentized plant hormones [4, 13-16]; its methods are well standardized. A weakness is that homeopathic studies on plants sometimes yield contradictory results, for example, stimulation of growth in one and inhibition of growth in another laboratory, both findings being homogeneous and statistically significant within themselves [5-8]. One of the tasks of fundamental homeopathy research must be to better define the conditions (methodological, seasonal, and geographic) which produce such consistent, yet contradictory results.

It may here be referred to a project (1989-2009) on amphibian metamorphosis under the influence of dilutions of thyroxin [10-12]. When in experiments special highland amphibian were used, effects of extremely diluted agitated probes added to the basin water (30x, decrease of metamorphosis speed) were independently found in 9 out of 10 studies performed by different researchers at sites including various university laboratories [13]. In contrast, animals from lowland biotopes obviously did not react to thyroxin 30x [12]. The project helped to highlight pitfalls and challenges in high dilution research [20, 21].

The authors interpret the 2008-2009 data as being in line with the 2007 findings [4], that is, as confirmation that gibberellic acid 30x does influence stalk growth. This would further confirm the hypothesis 
that information can be stored in the test liquid [4, 18, 22, 23], even at a dilution of the original substance beyond Avogadro's value; and that the wheat bioassay [4] is sensitive to such information. It also was established that outcomes to this effect are best obtained in the autumn season, that is, that experiments should be performed in autumn season. Further research work to explore time-related dependencies could include other seasons of the year.

In order to facilitate manageability of the experimental setup, further experiments on wheat and gibberellic acid were performed on germination within one day [24].

\section{DISCLOSURE}

The paper presented here is part of a doctoral thesis.

\section{REFERENCES}

[1] L. Kolisko, Physiologischer und Physikalischer Nachweis der Wirksamkeit kleinster Entitäten bei Sieben Metallen, Goetheanum, Dornach, Switzerland, 1926.

[2] W. Pongratz and P. C. Endler, "Reappraisal of a classical botanical experiment in ultra high dilution research," in Ultra High Dilution: Physiology and Physics, P. C. Endler and J. Schulte, Eds., pp. 121-128, Kluwer Academic Publishers, Dodrecht, The Netherlands, 1994.

[3] W. Pongratz, A. Nograsek, and P. C. Endler, "Highly diluted agitated silver nitrate and wheat seedling development," in Fundamental Research in Ultra High Dilution and Homoeopathy, J. Schulte and P. C. Endler, Eds., pp. 143-152, Kluwer Academic Publishers, Dodrecht, The Netherlands, 1998.

[4] A. Pfleger, J. Hofäcker, W. Scherer-Pongratz, H. Lothaller, C. Reich, and P. C. Endler, "The effect of extremely diluted agitated gibberellic acid (10e-30) on wheat seedling development-a two researcher study," Complementary Therapies in Medicine, vol. 19, pp. 164-169, 2011.

[5] L. Betti, M. Brizzi, D. Nani, and M. Peruzzi, "Effect of high dilutions of Arsenicum album on wheat seedlings from seed poisoned with the same substance," British Homoeopathic Journal, vol. 86, pp. 86-89, 1997.

[6] M. Brizzi, D. Nani, M. Peruzzi, and L. Betti, "Statistical analysis of the effect of high dilutions of arsenic in a large dataset from a wheat germination model," British Homeopathic Journal, vol. 89, no. 2, pp. 63-67, 2000.

[7] M. Binder, S. Baumgartner, and A. Thurneysen, "The effects of a 45x potency of Arsenicum album on wheat seedling growth - a reproduction trial," Forschende Komplementarmedizin, vol. 12, no. 5, pp. 284-291, 2005.

[8] D. Nani, M. Brizzi, L. Lazzarato, and L. Betti, "The role of variability in evaluating ultra high dilution effects: considerations based on plant model experiments," Forschende Komplementarmedizin, vol. 14, no. 5, pp. 301305, 2007.

[9] P. C. Endler, A. Pfleger, K. Thieves, T. Reischl, and C. Reich, "Proposal for a comparison on relative differences in fundamental botanical homeopathy research," European Journal of Integrative Medicine, vol. 1, no. 4, p. 246, 2009.

[10] P. C. Endler, W. Pongratz, G. Kastberger, F. A. C. Wiegant, and J. Schulte, "The effect of highly diluted agitated thyroxine on the climbing activity of frogs," Veterinary and Human Toxicology, vol. 36, no. 1, pp. 56-59, 1994.

[11] C. Zausner, H. Lassnig, P. C. Endler et al., "Die wirkung von "homöopathisch" zubereitetem thyroxin auf die metamorphose von hochlandamphibien—ergebnisse einer multizentrischen kontrollstudie," Perfusion, vol. 17, pp. 268-276, 2002.

[12] P. C. Endler, W. Scherer-Pongratz, G. Lingg, and H. Lothaller, "Amphibian metamorphosis and a reverse effect of homeopathically prepared thyroxin—studies 1990-2010," Journal of Biosciences. In press.

[13] S. Baumgartner, A. Thurneysen, and P. Heusser, "Growth stimulation of dwarf peas (Pisum sativum L.) through homeopathic potencies of plant growth substances," Forschende Komplementarmedizin, vol. 11, no. 5, pp. 281292, 2004.

[14] S. Baumgartner, D. Shah, J. Schaller, U. Kämpfer, A. Thurneysen, and P. Heusser, "Reproducibility of dwarf pea shoot growth stimulation by homeopathic potencies of gibberellic acid," Complementary Therapies in Medicine, vol. 16, no. 4, pp. 183-191, 2008. 
[15] T. Jäger, C. Scherr, M. Simon, P. Heusser, and S. Baumgartner, "Effects of homeopathic Arsenicum album, nosode, and gibberellic acid preparations on the growth rate of arsenic-impaired duckweed (Lemna gibba L.)," TheScientificWorldJOURNAL, vol. 10, pp. 2112-2129, 2010.

[16] B. Hamman, G. Koning, and K. H. Lok, "Homeopathically prepared gibberellic acid and barley seed germination," Homeopathy, vol. 92, no. 3, pp. 140-144, 2003.

[17] B. Stock-Schröer, H. Albrecht, L. Betti et al., "Reporting experiments in homeopathic basic research (REHBaR)—a detailed guideline for authors," Homeopathy, vol. 98, no. 4, pp. 287-298, 2009.

[18] P. Endler, K. Thieves, C. Reich et al., "Repetitions of fundamental research models for homeopathically prepared dilutions beyond 10e-23: a bibliometric study," Homeopathy, vol. 99, no. 1, pp. 25-36, 2010.

[19] C. Endler, Name the 2010 Breakthrough, 2011, http://www.sciencemag.org/.

[20] P. C. Endler, Homeopathy Research-An Expedition Report. An Old Healing System Gains Plausibility, Maudrich, Graz, Austria, 2003.

[21] P. C. Endler, Expedition Homöopathieforschung. Ein Altes Heilsystem Wird Plausibel, Maudrich, Vienna, Austria, 2006.

[22] L. Montagnier, "Newsmaker interview: luc montagnier. French Nobelist escapes 'intellectual terror' to pursue radical ideas in China. Interview by Martin Enserink," Science, vol. 330, no. 6012, p. 1732, 2010.

[23] B. Marschollek, M. Nelle, M. Wolf, S. Baumgartner, P. Heusser, and U. Wolf, "Effects of exposure to physical factors on homeopathic preparations as determined by ultraviolet light spectroscopy," TheScientificWorldJOURNAL, vol. 10, pp. 49-61, 2010.

[24] H. Hartung, S. Schiestl, W. Matzer, and P. C. Endler, "Wheat germination (20 hrs) and extremely diluted gibberellic acid (10e-30). Explorative experiments on a fundamental homoeopathy research model," European Journal of Integrative Medicine, vol. 2, pp. 224-245, 2010.

\section{This article should be cited as follows:}

Peter Christian Endler, Wolfgang Matzer, Christian Reich, Thomas Reischl, Anna Maria Hartmann, Karin Thieves, Andrea Pfleger, Jürgen Hoföcker, Harald Lothaller, and Waltraud Scherer-Pongratz, "Seasonal Variation of the Effect of Extremely Diluted Agitated Gibberellic Acid (10e-30) on Wheat Stalk Growth: A Multiresearcher Study,” TheScientific WorldJOURNAL, vol. 11, pp. 1667-1678, 2011. 

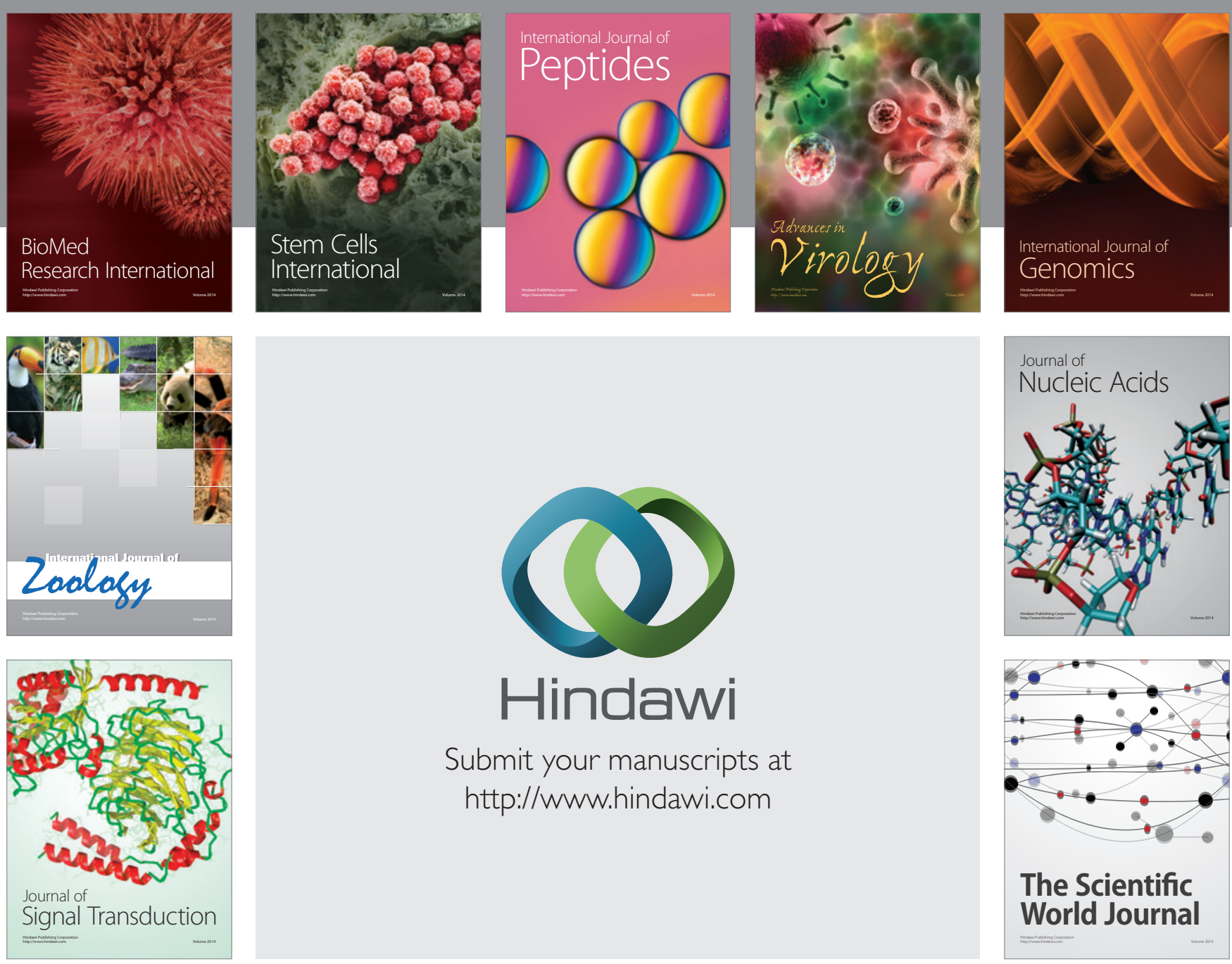

Submit your manuscripts at

http://www.hindawi.com
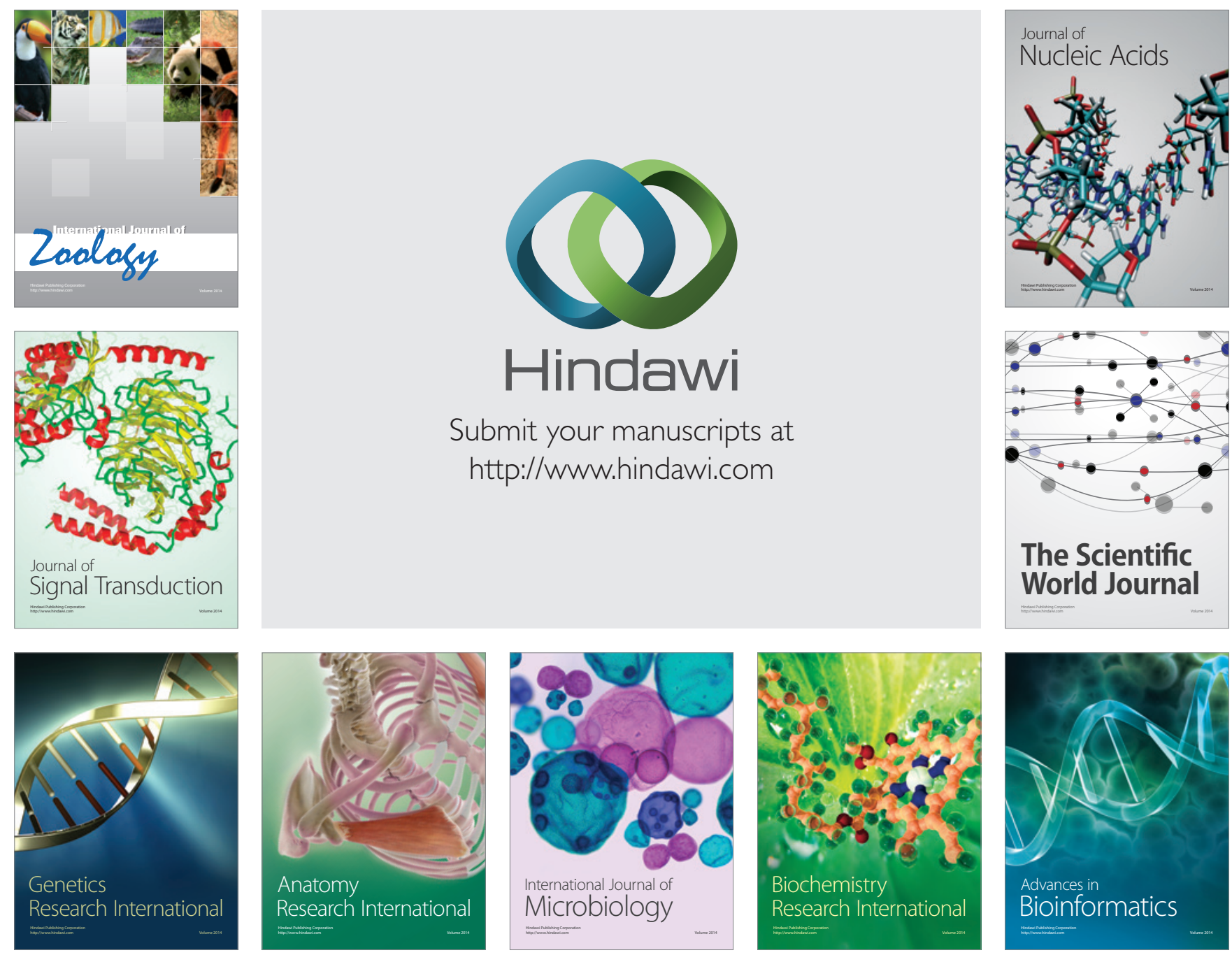

The Scientific World Journal
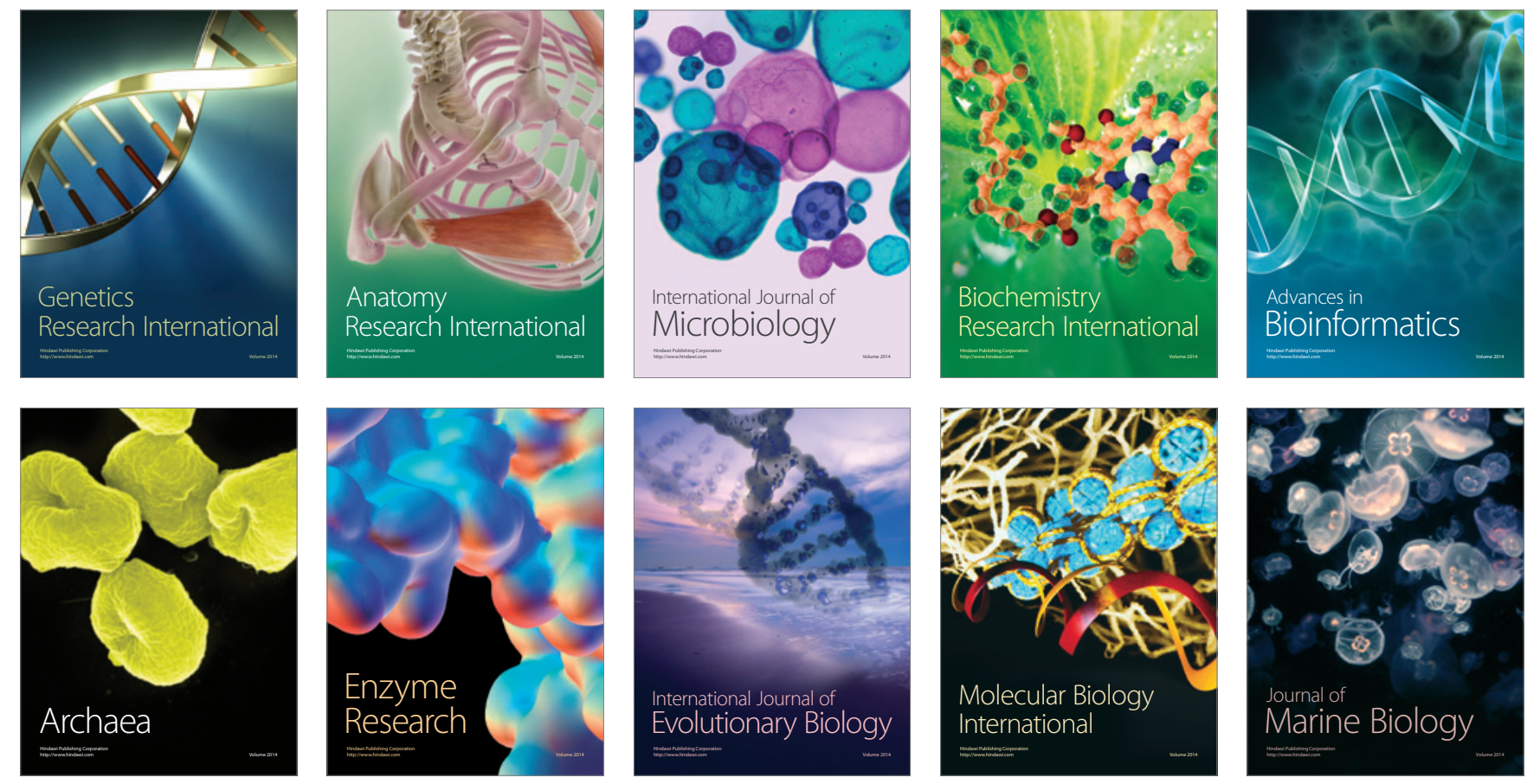